\title{
Understanding internetwork magnetic fields as determined from visible and infrared spectral lines
}

\author{
L. R. Bellot Rubio ${ }^{1}$ and M. Collados ${ }^{2}$ \\ 1 Kiepenheuer-Institut für Sonnenphysik, Schöneckstr. 6, 79104 Freiburg, Germany \\ 2 Instituto de Astrofísica de Canarias, 38200 La Laguna, Tenerife, Spain
}

Received 25 February 2003 / Accepted 7 May 2003

\begin{abstract}
We present numerical experiments aimed at understanding why near-infrared observations systematically deliver weak magnetic fields in the internetwork, whereas analyses based on visible lines indicate that $\mathrm{kG}$ fields are ubiquitous. Synthetic noisy Stokes $V$ profiles of the iron lines at $6302 \AA$ and $1.565 \mu \mathrm{m}$ have been produced under varying conditions in an effort to simulate polarized spectra coming from the internetwork. An inversion technique has been applied to the profiles, as it is usually done with real observations, in order to derive the distribution of magnetic fields in the simulated region. Our results show that infrared lines yield distributions which are very similar to those used as input for the simulation, while visible lines are to a large extent affected by noise. Analyses based on the Fe I lines at $6302 \AA$ may lead to an overabundance of $\mathrm{kG}$ fields if the signal-to-noise ratio in Stokes $V$ is poorer than about 10. A particular example is shown where strong fields are retrieved in nearly $30 \%$ of the pixels of a simulated internetwork region in which only fields of $200 \mathrm{G}$ exist.
\end{abstract}

Key words. polarization - line: profiles - Sun: infrared - Sun: photosphere - Sun: magnetic fields

\section{Introduction}

Internetwork (IN) magnetic fields have attracted the interest of solar physicists during the past few years (see, e.g., the review by Steiner 2003). One reason for that is the suspicion that they may be responsible for a significant fraction of the total magnetic flux of the sun. Another reason is that they hold the key to decide whether the magnetism of the quiet photosphere is controlled by a local dynamo (e.g., Emonet \& Cattaneo 2001 and references therein) or by other processes like, for example, the decay of active regions. Since the magnetic field distribution at the surface is an expression of the relevant physical mechanism, observational determinations of the field strength distribution (in terms of probability density functions, or PDFs) are important to distinguish between scenarios. The internetwork is preferred for this characterization because the $\mathrm{kG}$ fields occurring in facular and network regions are believed to be produced by other processes, most notably convective collapse (Parker 1978; see also Solanki et al. 1996; and Bellot Rubio et al. 2001).

From an observational point of view, the determination of magnetic field PDFs in the internetwork is plagued with difficulties. The IN polarization signals are very small because the magnetic field occupies a tiny fraction of the resolution element. As a consequence, they are easily hidden by noise. Cancellation of Stokes $V$ signals due to mixed polarities in the

Send offprint requests to: L. R. Bellot Rubio, e-mail: lbellot@kis.uni-freiburg.de resolution element may also be important. Socas-Navarro \& Sánchez Almeida (2002) and Khomenko et al. (2003), for example, report that $35 \%$ and $30 \%$ of the spatial points showing magnetic signal in their observed maps require mixed polarities. This explains why internetwork fields remain elusive. High sensitivity instruments are absolutely necessary for a reliable characterization of their properties. Current grating spectropolarimeters and narrow-band filters are just at the limit of the required sensitivity if typical exposure times are used.

The IN magnetic field PDFs determined so far are contradictory (cf. Steiner 2003). Analyses based on visible lines suggest that the internetwork contains a sizeable fraction of fields in the $\mathrm{kG}$ range (Grossmann-Doerth et al. 1996; Sánchez Almeida \& Lites 2000; Socas-Navarro \& Sánchez Almeida 2002; Domínguez Cerdeña et al. 2003; but see Lites 2002). Analyses based on infrared spectral lines, on the contrary, indicate that strong fields are very rare and that weak fields dominate the PDF (Lin 1995; Collados 2001; Khomenko et al. 2003). The particular scenario favored by each author does not seem to depend on the data employed (spectrograph or narrow-band filter observations), on the way the PDF is obtained (through Gaussian fitting, Stokes $V$ amplitude fitting, or profile inversion), or on the assumptions regarding the model atmosphere (one or more components in the resolution element), but only on the wavelength of the spectral lines utilized. Several explanations have been offered to understand these conflicting results. Part of the difference could be due to network $(\mathrm{kG})$ elements being present in the region under 
analysis. This is probably the reason why the visible observations of Grossmann-Doerth et al. (1996) revealed so many strong fields. Other explanations are based on the different thermal and magnetic sensitivity of infrared and visible lines (Sánchez Almeida \& Lites 2000), although it is not clear whether the proposed mechanisms are model-dependent or not. Changes in the separation of the Zeeman components (and hence the saturation of the polarization signal) start to be noticeable in Stokes $V$ for field strengths as low as $300-500 \mathrm{G}$ in the case of infrared lines and as high as $1000 \mathrm{G}$ in the case of visible lines. The different field strengths at which the transition from weak-field to strong-field behavior takes place have been used to suggest that, if several magnetic fields coexist in the resolution element, visible observations would tend to detect the stronger fields, whereas infrared lines would favor the weaker fields (Socas-Navarro 2003).

Here we argue that noise is essential for understanding the differences between the PDFs retrieved from visible and nearinfrared Stokes profiles. Our suggestion is based on the results of a numerical experiment. Synthetic Stokes $V$ profiles of the Fe I lines at $6302 \AA$ and $1.565 \mu \mathrm{m}$ emerging from a simulated internetwork region have been generated under varying conditions. After adding noise, the visible and infrared profiles have been inverted separately in terms of a single magnetic component. This allows us to investigate the properties of the resulting PDFs, and to assess how they are influenced by the noise.

\section{Simulation of Stokes profiles}

We synthesize the Stokes $V$ profiles of the Fe I lines at 6301, 6302, 15648 and $15652 \AA$ emerging from internetwork regions. There is growing evidence, both from observations and from numerical simulations, that magnetic fields are ubiquitous in the internetwork and that all field strengths from zero to the thermal equipartition value of about $1.5 \mathrm{kG}$ are possible. This implies that magnetic fibrils of various strengths may be present in the resolution element at moderate $(\sim 1$ arcsec $)$ angular resolution. We include these ingredients in our simulations by considering that the polarization signals emerging from each resolution element are produced by two spatially separated magnetic fibrils of different field strengths. The number of magnetic components is restricted to two for simplicity, but we note that having more components would not change the results qualitatively. The magnetic field strength of the two atmospheres is assumed to be constant with optical depth. The temperature stratifications are taken to be that of the quiet sun (represented by the Harvard Smithsonian Reference Atmosphere of Gingerich et al. 1971). A fraction $\alpha$ of the resolution element is magnetized, with the first component contributing a fraction $\beta$ and the second the remaining $1-\beta$. Thus, the emergent Stokes $V$ profiles are computed as $V=\alpha\left[\beta V_{1}+(1-\beta) V_{2}\right]$, where $V_{i}$ represents the signal due to component $i$. The magnetic filling factor $\alpha$ is an important quantity in determining the amplitude of the simulated Stokes $V$ signal.

In order to mimic real observations, white noise is added to the profiles at the level of $10^{-3}$ and $10^{-4}$ of the continuum intensity $I_{\mathrm{c}}$. The interpretation of the resulting circular polarization profiles is done by assuming that there is only one single magnetic component in the resolution element. This is a common assumption in analyses of the internetwork. The Stokes $V$ profiles are subject to two inversions (one for the visible lines and other for the infrared lines) in order to determine the parameters of the model atmosphere that provides the best fit to the observations. To this end, we use the SIR code developed by Ruiz Cobo \& del Toro Iniesta (1992). SIR iteratively modifies a guess model atmosphere by means of a nonlinear, least-squares algorithm until the synthetic profiles match the observed ones. Depending on the application, the user decides which atmospheric parameters are to be determined. In our case, free parameters of the inversion are the magnetic field strength, the magnetic filling factor, and the macroturbulent velocity. The macroturbulence is allowed to vary in order to provide a means to account for the broadening of the Stokes $V$ profiles induced by the existence of two magnetic fields, which cannot be explained in terms of a single magnetic component. This additional broadening is very noticeable in the infrared lines, but not in the visible lines.

\section{Results}

Let us assume that the actual distribution of magnetic fields in the internetwork follows the law $p(B)=C \exp \left(-B / B_{0}\right)$, where $p(B) \mathrm{d} B$ is the probability of occurrence of magnetic fields with strengths from $B$ to $B+\mathrm{d} B$, and $C$ is a normalization constant. We take $B_{0}=250 \mathrm{G}$ as indicated by the infrared measurements of Collados (2001) and Khomenko et al. (2003).

The simulation starts by extracting at random two magnetic fields from the actual distribution $p(B)$. We then compute the corresponding Stokes $V$ signals, and combine them using a value for $\beta$. In accordance with recent observational results, we assume that the magnetic atmospheres occupy a fraction $\alpha=0.05$ of the resolution element. As many as 10000 simulated profiles have been produced in this way. The magnetic field distributions inferred from the inversion of such profiles are presented in the following sections for two values of $\beta$.

\subsection{Case $\beta=0$}

The situation in which only one magnetic field is present in the resolution element can be modeled with $\beta=0$. This case is considered here because it represents the simplest scenario one can imagine for the internetwork.

Figure 1 shows the field strength distributions inferred from the visible and infrared lines. When the noise is small (upper panel), the PDF determined using the infrared lines is very similar to the original distribution. By contrast, the PDF resulting from the visible lines shows a pronounced deficit of weak fields below $400 \mathrm{G}$. With larger noise at the level of $10^{-3} I_{\mathrm{c}}$ (lower panel), the infrared lines still do an excellent job in recovering the original PDF down to field strengths of about $350 \mathrm{G}$. However, the PDF delivered by the visible lines bears little resemblance with the true PDF. The most prominent feature of the distribution is the peak at about $900 \mathrm{G}$, with very broad wings, especially towards lower field strengths. Compared with the original PDF, there is an excess of strong fields and 

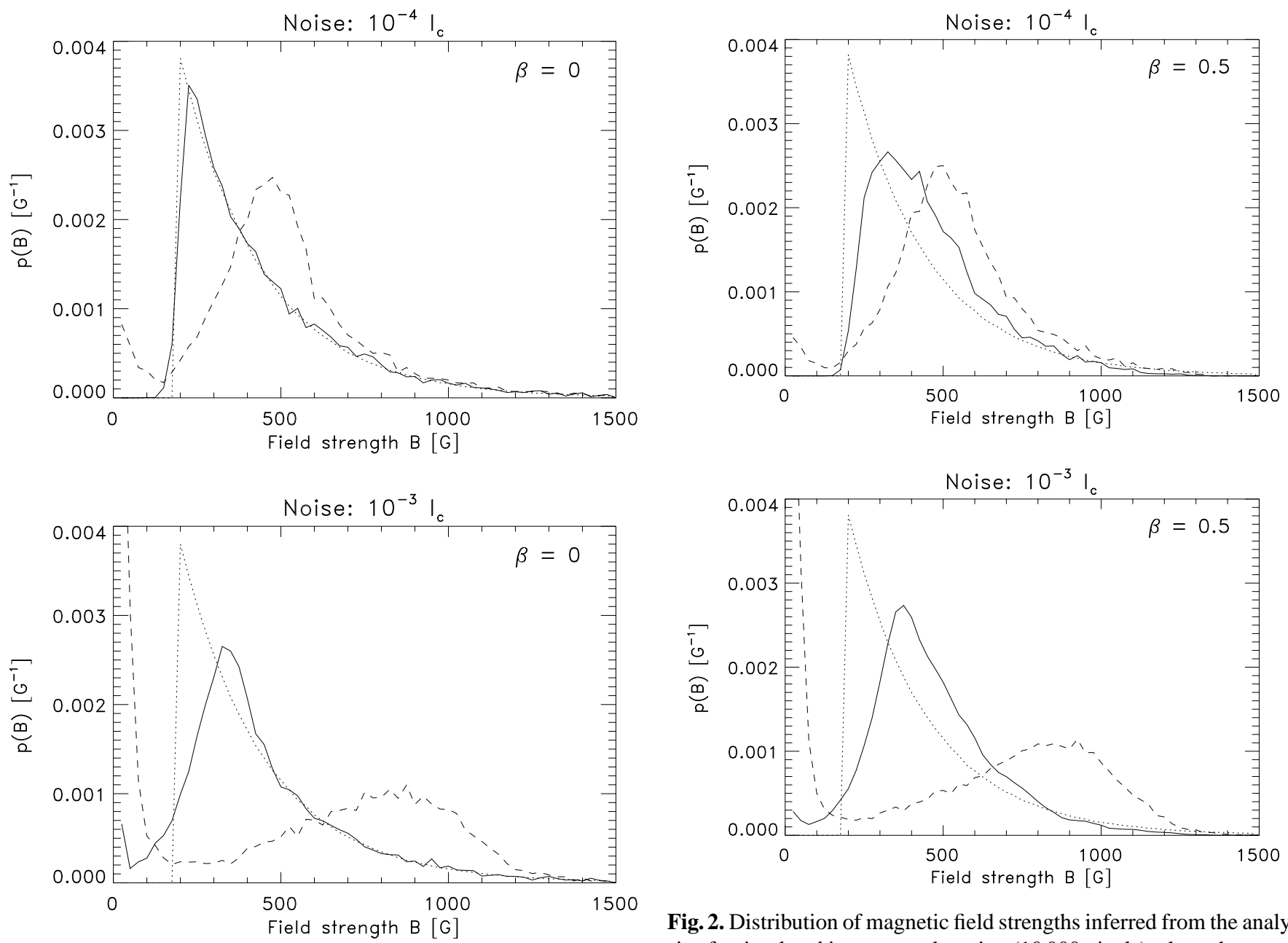

Fig. 1. Distribution of magnetic field strengths inferred from the analysis of a simulated internetwork region (10000 pixels) where the actual distribution is $p(B)=C \exp (-B / 250)$ and each resolution element contains one single magnetic field $(\beta=0)$. Solid and dashed lines give the PDFs resulting from the infrared and the visible lines, respectively. The dotted line represents the original PDF. The binsize is 25 G. Top: Noise added to Stokes $V$ at the level of $10^{-4}$ of the continuum intensity $I_{\mathrm{c}}$. Bottom: Noise at the level of $10^{-3} I_{\mathrm{c}}$.

a significant deficit of weak fields larger than 200 G. Indeed, most pixels are interpreted as being characterized by field strengths smaller than $100 \mathrm{G}$ or larger than $800 \mathrm{G}$. Thus, one would conclude from the visible PDF that the simulated IN region contains a sizeable fraction of $\mathrm{kG}$ fields, while in reality there are very few strong fields in it.

\subsection{Case $\beta=0.5$}

The situation in which there are two magnetic fields in the same resolution element is modeled here by setting $\beta=0.5$, i.e., both fields contribute to the Stokes $V$ profile with the same weight. In the absence of any observational result concerning the dependence of the magnetic filling factor on field strength in internetwork regions, the choice of $\beta=0.5$ seems appropriate.

Figure 2 shows the PDFs resulting from the visible and infrared lines in this case. Despite the different number of

Fig. 2. Distribution of magnetic field strengths inferred from the analysis of a simulated internetwork region (10000 pixels) where the actual distribution is $p(B)=C \exp (-B / 250)$ and two magnetic fibrils coexist in each resolution element with $\beta=0.5$. Solid and dashed lines represent the PDFs resulting from the infrared and the visible lines, respectively. The dotted line gives the original field strength distribution. Top: Noise added at the level of $10^{-4} I_{\mathrm{c}}$. Bottom: Noise at the level of $10^{-3} I_{\mathrm{c}}$.

magnetic components in the resolution element, the new PDFs are very similar to those presented in Fig. 1. The only difference is that the peaks are slightly broader and more shifted towards stronger fields. Independently of the noise, the infrared lines deliver magnetic field PDFs which are in reasonable accord with the actual distribution. Indeed, above $400 \mathrm{G}$ the infrared PDFs are well described by an exponential law of the form $p(B) \propto \exp (-B / 250)$. The visible lines, however, are very much affected by the noise. As the noise level increases, the peak of the distribution moves to stronger fields. The maximum of the PDFs determined from the visible lines occurs at $500 \mathrm{G}$ with noise of $10^{-4} I_{\mathrm{c}}$, and at $900 \mathrm{G}$ with noise of $10^{-3} I_{c}$. Again, one would conclude that a significant fraction of pixels in the simulated region contains strong fields. This is true for noise levels of about $10^{-3} I_{\mathrm{c}}$ (typical of current spectropolarimetric observations). Much lower noise levels, at least by a factor of ten, would be needed for the visible lines to recover the true distribution of magnetic fields. 


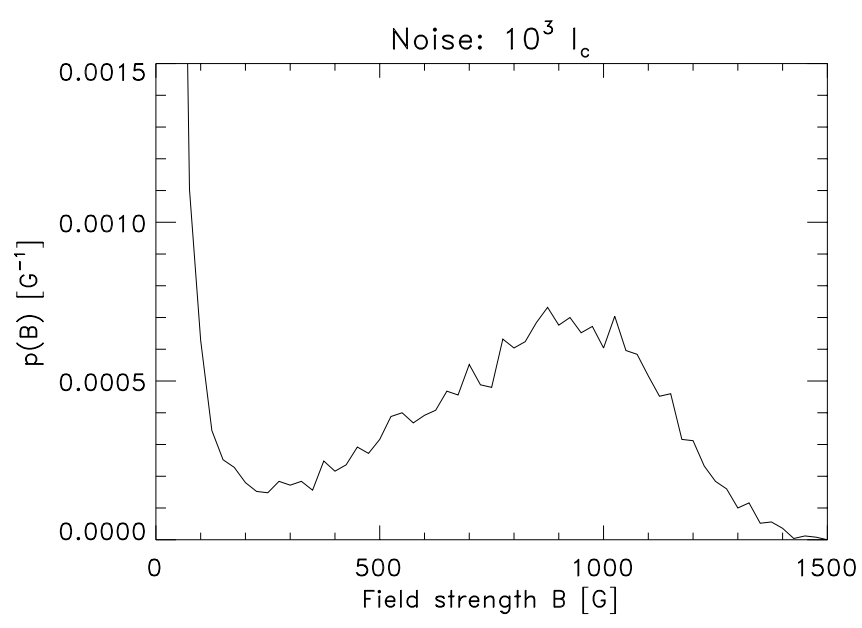

Fig. 3. Distribution of magnetic field strengths determined from an inversion of the Stokes $V$ profiles of the visible lines emerging from a simulated internetwork region where only fields of $200 \mathrm{G}$ are present. Noise of $10^{-3} I_{\mathrm{c}}$ has been added to the Stokes $V$ profiles. If the resulting PDF is taken at face value, $27 \%$ of the pixels would be associated with strong fields $(>800 \mathrm{G})$.

\section{Discussion}

The excess of strong fields indicated by the visible lines is particularly disturbing. In order to understand this behavior, we have repeated the analysis assuming that all field strengths in the simulated internetwork region are equal to $200 \mathrm{G}$, i.e., no strong fields exist. The results of this experiment, carried out with noise in the profiles at the level of $10^{-3} I_{\mathrm{c}}$ and $\alpha=0.05$, are summarized in Fig. 3. Again, the visible lines yield a peak of strong fields at around $0.9 \mathrm{kG}$, with most of the pixels being assigned field strengths of less than $100 \mathrm{G}$. Taken at face value, the retrieved PDF indicates that $27 \%$ of the pixels contain $\mathrm{kG}$ fields. This leads us to conclude that noise is capable of modifying the polarization signals in such a way that the inversion code interprets them as being caused by strong fields. At high noise levels, the small changes in the Zeeman splitting of the visible lines cannot be used as a diagnostics, and the ratio of Stokes $V$ amplitudes of the two lines is severely affected. As a result, both strong and weak fields are compatible with the observations. Figure 4 shows one such example. The observed profile has been synthesized using a magnetic field of $200 \mathrm{G}$ and noise of $10^{-3} I_{\mathrm{c}}$. The inversion of this profile suggests a magnetic field of $1250 \mathrm{G}$. The Stokes $V$ signal corresponding to such a strong field is given by the thick solid line. In view of the quality of the fit, one would never say that the inferred value of $1250 \mathrm{G}$ is erroneous. Only when the best-fit profile is compared with the original Stokes $V$ spectrum without noise (the smooth thin solid line of Fig. 4) do differences in the amplitude and position of the peaks become apparent.

We may expect the situation to improve as the SNR of the observations increases. At low noise levels, the different Zeeman splittings and Stokes $V$ amplitude ratios induced by strong and weak fields cannot be confused any more. Since the inversion code has much more information, the derived field strengths become closer to the real values. This is demonstrated in Fig. 5, where the peak of the PDF determined from

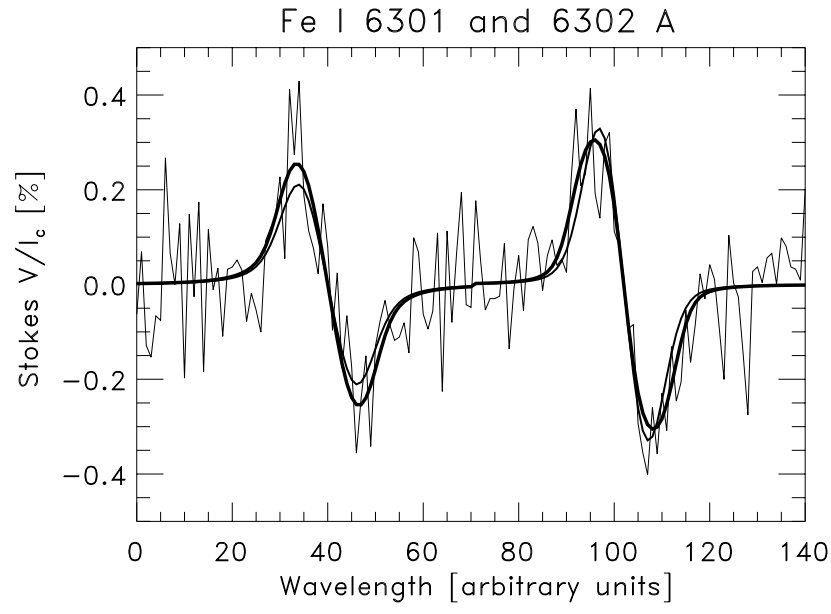

Fig. 4. Stokes $V$ signal simulated with a magnetic field strength of $200 \mathrm{G}, \alpha=0.05$, and noise at the level of $10^{-3} I_{\mathrm{c}}$ (thin solid line). For this particular realization of the noise, the best-fit profile (thick solid line) indicates a field strength of $1250 \mathrm{G}$. The smooth thin solid line is the original spectrum without noise.

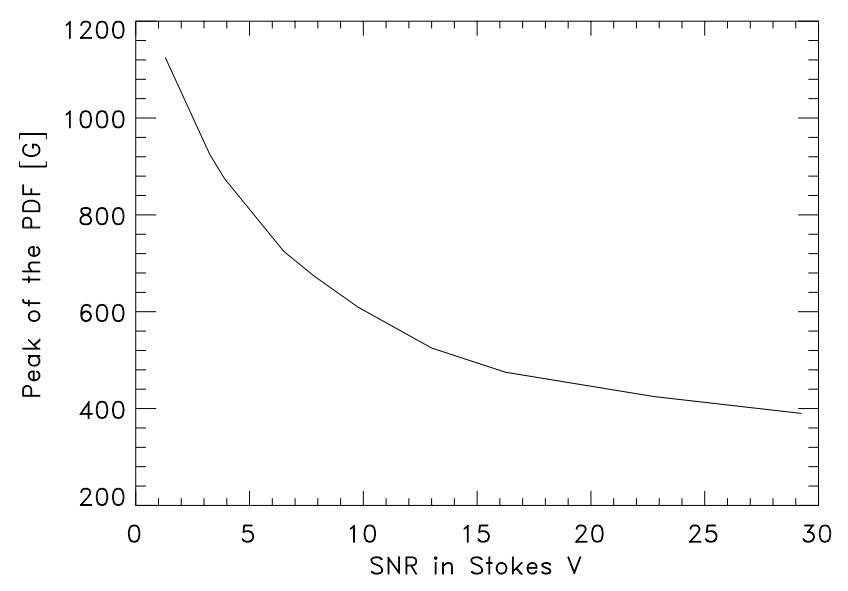

Fig. 5. Position of the peak of the PDF inferred from the visible lines as a function of the signal-to-noise ratio in Stokes $V$. Only fields of $200 \mathrm{G}$ are present in the original distribution.

the inversion of the visible lines is plotted as a function of the signal-to-noise ratio (SNR) in Stokes $V^{1}$. As before, we assume that all magnetic fields in the simulated region are equal to $200 \mathrm{G}$. In order to produce Fig. 5, different noises have been added to the profiles, leading to SNRs in Stokes $V$ in the range 1-30. Strictly speaking, Fig. 5 represents only the case in which all magnetic fields have strengths of $200 \mathrm{G}$. However, it should be valid also for any field weaker than about $1 \mathrm{kG}$. The curve in Fig. 5 slowly converges to $200 \mathrm{G}$, but for

\footnotetext{
${ }^{1}$ For a given noise level $N$, the SNR in Stokes $V$ is always smaller than in Stokes $I$ because the circular polarization signal is smaller than the intensity signal. As an example, take $N=10^{-3} I_{\mathrm{c}}$ and $V_{\max } / I_{\mathrm{c}} \sim$ $3 \times 10^{-3}$ (these values correspond to the profiles depicted in Fig. 4). The SNR in the continuum intensity is $S / N=I_{\mathrm{c}} /\left(10^{-3} I_{\mathrm{c}}\right)=1000$. The SNR in Stokes $V$ is only $3 \times 10^{-3} I_{\mathrm{c}} /\left(10^{-3} I_{\mathrm{c}}\right) \sim 3$. The minimum detectable polarization signal (the polarimetric sensitivity) is usually estimated from the noise of the observations. However, the important quantity determining the reliability of the inferred magnetic field is the SNR in Stokes $V$.
} 

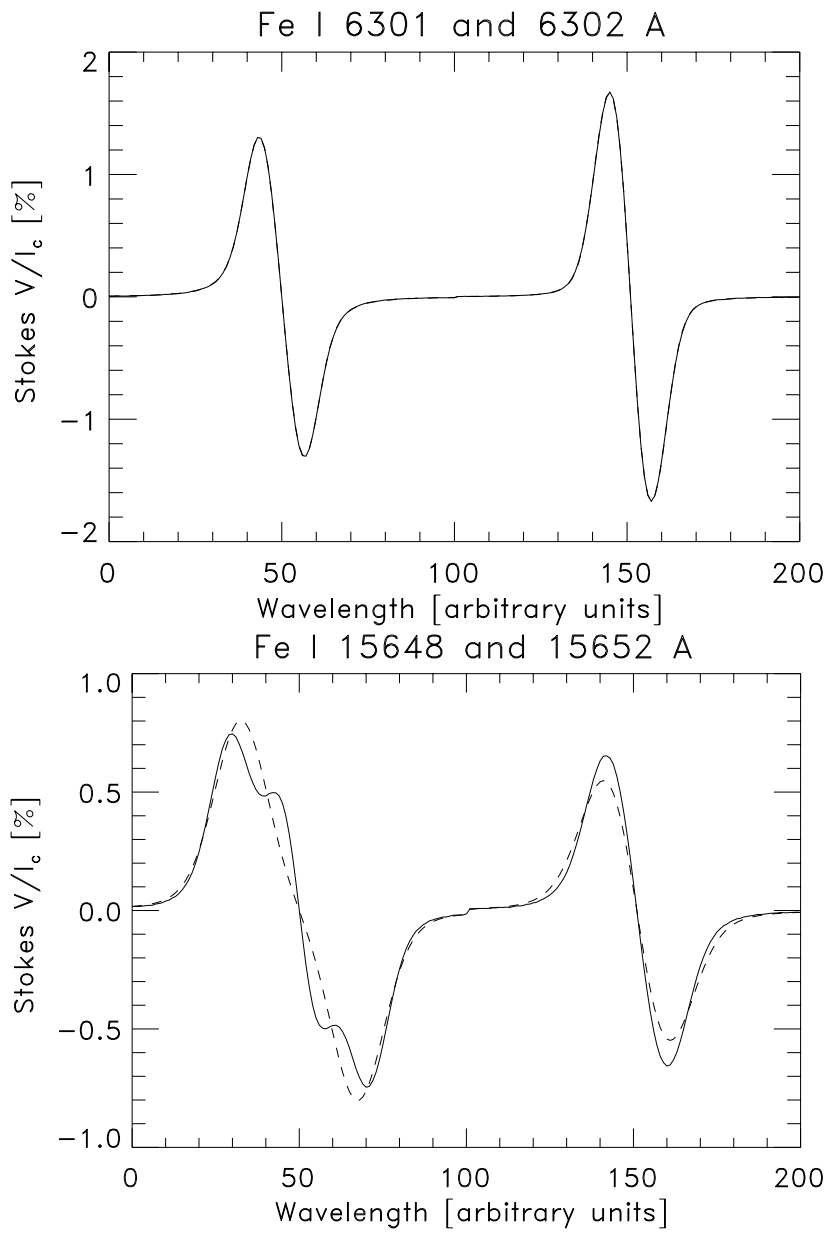

Fig. 6. Top: simulated (solid line) and best-fit (dashed line) profiles of the visible lines for $\alpha=1$ and $\beta=0.5$. The fit is so good that the dashed line is indistinguishable. Bottom: same for the infrared lines.

typical noise levels the maximum of the PDF occurs at much stronger fields. Indeed, a minimum SNR of 10 is required in the Stokes $V$ signal of the Fe I $6302.5 \AA$ line if the PDF is not to peak above $600 \mathrm{G}$. Adopting reasonable numbers for the polarization signal emerging from the internetwork, this limit corresponds to SNRs of roughly $5-10 \times 10^{3}$ in the continuum intensity.

The most important conclusion that can be drawn from these experiments is that internetwork magnetic field PDFs resulting from visible lines are very sensitive to noise in the profiles. Empirically determined PDFs showing maxima at large field strengths should be regarded with suspicion, especially if short exposure times were used during the observations.

Another interesting outcome of the numerical experiments presented in Sect. 3 is the fact that the PDFs retrieved from the infrared lines for the cases $\beta=0$ and $\beta=0.5$ are essentially the same down to about $400 \mathrm{G}$. This implies that the original distribution is recovered in a statistical sense independently of whether the resolution elements are occupied by one or more magnetic components. In order to understand this result, let us consider the simple situation in which two magnetic components having field strengths of 200 and $1200 \mathrm{G}$ coexist in the

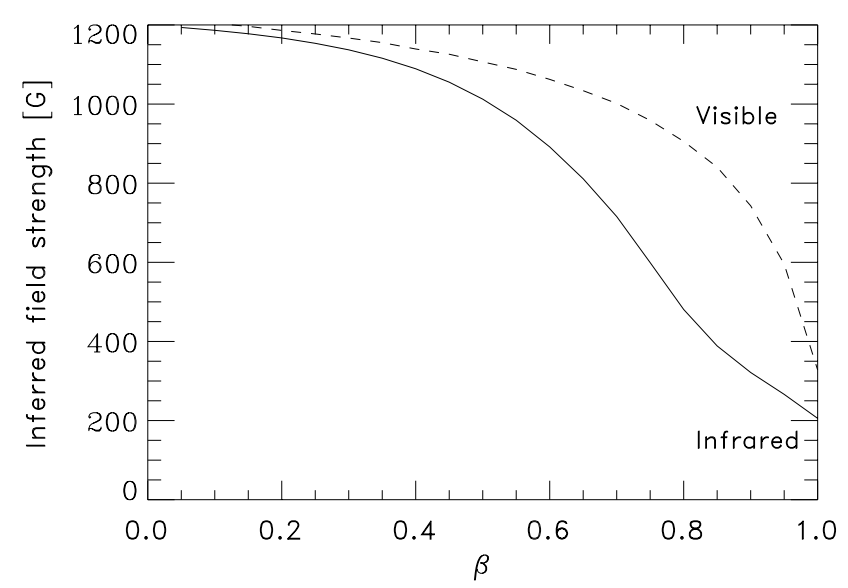

Fig. 7. Field strengths inferred from the fit of simulated profiles assuming that they are produced by one magnetic atmosphere, as a function of $\beta$ (the fraction of magnetized area covered by weak fields). The Stokes $V$ spectra have been constructed by mixing the signals of two atmospheres having magnetic fields of $200 \mathrm{G}$ and $1200 \mathrm{G}$.

resolution element and the filling factor of the weak field component, $\beta$, varies from 0 to 1 . The two limiting cases correspond to only strong or weak fields being present in the resolution element. For simplicity, no noise is added to the profiles.

Figure 6 displays, as an example, the Stokes $V$ profiles of the visible and infrared lines when $\beta=0.5$. The visible lines and Fe I $15652 \AA$ show no obvious trace of two different magnetic fields due to the small Zeeman splittings. This is not the case for the infrared Fe I line at $15648 \AA$. With a Landé factor of 3 , the signals generated by the strong and weak field components are clearly visible due to the huge splitting of the former. The dashed lines give the best-fit profiles resulting from the inversion. As can be seen, the fit is excellent for the visible lines and somewhat worse for the infrared lines. If one magnetic atmosphere is used to interpret the Stokes $V$ signal of Fe I $15648 \AA$, the inferred field strength lies somewhere in between the original 200 and $1200 \mathrm{G}$ in order to have an intermediate Zeeman splitting. The Stokes $V$ amplitude of Fe I $15652 \AA$ induced by such a field is too small, as can be seen in the lower panel of Fig. 6. The misfit could be removed by increasing the field strength, but this would lead to a much worse fit to Fe I $15648 \AA$. The best compromise is then an intermediate value which fits equally well (or worse) the Zeeman splitting of Fe I $15648 \AA$ and the Stokes $V$ amplitude of $15652 \AA$. Hence, if two components are present in the resolution element, we may expect that the field strength determined from the infrared lines will not correspond to any of the original fields unless the fraction of area occupied by one of them is very large.

This intuitive idea is confirmed in Fig. 7, where the field strengths estimated from the visible and infrared lines are plotted as a function of $\beta$. The visible lines deliver strong magnetic fields up to $\beta \sim 0.8$, whereas the infrared lines return all field strengths from $1200 \mathrm{G}$ to $200 \mathrm{G}$ as $\beta$ varies from 0 to 1 . Visible lines favor strong fields because the signal due to the weak field component is about five times smaller than that of the strong field, hence it does not contribute significantly to the 
total profile until $\beta$ is relatively large. For $\beta \lesssim 0.8$, the ratio of Stokes $V$ amplitudes as well as the Zeeman splitting of the visible lines indicate strong fields, and this is what the inversion code returns. The infrared lines, on the other hand, deliver field strengths which are somewhere in between the true fields present in the resolution element. Of course, the assumption of one magnetic component precludes a more realistic description of the various fields that may contribute to the observed signals. In spite of that, the picture drawn from the infrared lines is correct in a statistical sense, because the true PDF is recovered when a large number of pixels (i.e., the whole field of view) is considered. Thus, the PDFs resulting from the interpretation of infrared observations in terms of one single magnetic component are probably valid even if several magnetic fields coexist in the resolution element.

\section{Summary and conclusions}

In this paper we have presented the results of Montecarlo simulations aimed at understanding the internetwork magnetic field distributions derived from visible and infrared spectral lines. Stokes $V$ profiles of the Fe I lines at $6302 \AA$ and $1.565 \mu \mathrm{m}$ have been synthesized assuming different magnetic components in the resolution element and different noise levels. The visible and infrared lines have been inverted separately in order to determine the magnetic field distributions of a simulated internetwork region. This strategy has allowed us to assess the range of validity of the resulting distributions.

Three main conclusions can be drawn from our numerical experiments:

- The infrared lines do a reasonably good job in retrieving the PDF used as input for the simulation down to a few hundred Gauss, where the weak field regime may be considered to take over. This is true even if several magnetic fibrils are present in the resolution element, giving strong support to the observational PDFs obtained by Lin (1995), Collados (2001), and Khomenko et al. (2003).

- Visible lines are very sensitive to noise when dealing with low-amplitude polarization signals such as those found in internetwork regions. The magnetic field leaves its imprint mainly in the amplitude of the circular polarization profiles. As a consequence, it cannot be determined reliably at high noise levels. A particular example has been presented where the inversion of spectra of visible lines with noise of $10^{-3} I_{\mathrm{c}}$ reveals strong fields in $30 \%$ of the spatial points, although only fields of $200 \mathrm{G}$ were used as input. Infrared lines are much less sensitive to noise because of the larger Zeeman splitting.

- Signal-to-noise ratios in Stokes $V$ better than about 10 are needed to be confident about $\mathrm{kG}$ fields inferred from visible lines. This limit corresponds to signal-to-noise ratios of $5-10 \times 10^{3}$ in the continuum intensity for the conditions prevailing in the internetwork.

In order to reconcile the results of analyses based on visible and infrared observations, some authors have suggested that weak and $\mathrm{kG}$ fields coexist in the internetwork, with the strong fields occupying a tiny fraction of the resolution element (otherwise the infrared lines would detect them easily). While such an scenario cannot be ruled out, we warn that noise is an important ingredient for understanding the internetwork magnetic field distributions determined observationally. Noise affects the measurement of intrinsic field strengths from very weak polarization signals, especially in the visible. This may well explain the different magnetic field distributions derived from visible and infrared lines.

Our results suggest that internetwork magnetic field studies would greatly benefit from lower noise levels. Until larger solar telescopes become operational, the obvious way to improve the SNR of spectropolarimetric measurements is to use longer effective exposure times.

Acknowledgements. Comments and suggestions by Héctor Socas-Navarro and Oskar Steiner are gratefully acknowledged. This work has been partially funded by the Deutsche Forschungsgemeinschaft and by the Spanish Ministerio de Ciencia y Tecnología through project AYA2001-1649.

\section{References}

Bellot Rubio, L. R., Rodríguez Hidalgo, I., Collados, M., Khomenko, E., \& Ruiz Cobo, B. 2001, ApJ, 560, 1010

Collados, M. 2001, ASP Conf. Ser., 236, 255

Domínguez Cerdeña, I., Kneer, F., \& Sánchez Almeida, J. 2003, ApJ, 582, L55

Emonet, T., \& Cattaneo, F. 2001, ApJ, 560, L197

Gingerich, O., Noyes, R. W., Kalkofen, W., \& Cuny, Y. 1971, Sol. Phys., 18, 347

Grossmann-Doerth, U., Keller, C. U., \& Schuessler, M. 1996, A\&A, 315,610

Khomenko, E., Collados, M., Solanki, S. K., Lagg, A., \& Trujillo Bueno, J. 2003, A\&A, in press

Lin, H. 1995, ApJ, 446, 421

Lites, B. W. 2002, ApJ, 573, 431

Parker, E. N. 1978, ApJ, 221, 368

Ruiz Cobo, B., \& del Toro Iniesta, J. C. 1992, ApJ, 398, 375

Sánchez Almeida, J., \& Lites, B. W. 2000, ApJ, 532, 1215

Socas-Navarro, H. 2003, ASP Conf. Ser., in press

Socas-Navarro, H., \& Sánchez Almeida, J. 2002, ApJ, 565, 1323

Solanki, S. K., Zufferey, D., Lin, H., Rüedi, I., \& Kuhn, J. R. 1996, A\&A, 310, L33

Steiner, O. 2003, in Proc. NATO Advanced Research Workshop: Turbulence, Waves, and Instabilities in the Solar Plasma, ed. R. Erdelyi, \& K. Petrovay (Dordrecht: Kluwer), in press 